\title{
Test of Fibre Bragg Gratings samples under High Fast Neutrons Fluence
}

\author{
G. Cheymol, L. Remy, A. Gusarov, D. Kinet, P. Mégret, G. Laffont, T. Blanchet, A. Morana, E. Marin \\ and S. Girard Senior Member, IEEE
}

\begin{abstract}
Optical fibre sensors (OFS) are worthy of interest for measurements in nuclear reactor thanks to their unique features, particularly compact size and remote multi-point sensing for some of them. But besides non negligible constraints associated with the high temperature environment of the experiments of interest, it is well known that the performances of OFS can be severely affected by high level of radiations. The Radiation Induced Attenuation (RIA) in the fibre is probably most known effect, which can be to some extent circumvented by using rad hard fibres to limit the dynamic loss. However, when the fast neutron fluence reaches $10^{18}$ to $10^{19} \mathrm{n} / \mathrm{cm}^{2}$, the density and index variations associated to structural changes may deteriorate drastically the performances of OFS even if they are based on rad hard fibres, by causing direct errors in the measurements of temperature and/or strain changes. The aim of the present study is to access the effect of nuclear radiations on the Fabry Perot (FP) and of Fibre Bragg Grating (FBG) sensors through the comparison of measurements made on these OFS - or part of them - before and after irradiation [1]. In the context of development of OFS for high irradiation environment and especially for Material Testing Reactors (MTRs), Sake 2 experiment consists in an irradiation campaign at high level of gamma and neutron fluxes conducted on samples of fibre optics - bare or functionalised with FBG. The irradiation was performed at two levels of fast neutron fluence: 1 and $3.10^{19} \mathrm{n} / \mathrm{cm}^{2}$ $(\mathrm{E}>1 \mathrm{MeV})$, at $250^{\circ} \pm 25^{\circ} \mathrm{C}$, in the $\mathrm{SCK} \cdot \mathrm{CEN} \mathrm{BR} 2$ reactor (Mol Belgium). An irradiation capsule was designed to allow irradiation at the specified temperature without active control. The neutron fluence was measured with activation dosimeters and the results were compared with MCPN computations. Investigation of bare samples gives information on the density changes, while for the FBGs both density and refractive index perturbation are involved. Some results for bare fibres were reported recently. In this paper, we will focus on the measurements made on FBGs that have been manufactured by different laboratories on SMF 28 fibers: CEA, University of St-Etienne and University of Mons. Tested gratings have been written using various conditions (type of fibre, of laser, writing wavelength, power density, post writing thermal annealing,...), leading to various behaviours after Sake 2 irradiation. Bragg wavelength and reflectivity have been measured before and after irradiation thanks to a special mounting at the same temperature. It appears that a change in the
\end{abstract}

Manuscript received xx. This work was supported by French Nuclear Atomic Agency, Direction of Nuclear Energy, Nuclear Instrumentation project and SCK.CEN, Belgian Nuclear Research Center.

G. Cheymol, L. Remy, are with Den - Service d'Etudes Analytiques et de Réactivité des Surfaces (SEARS), CEA, Université Paris-Saclay, F-91191, Gif sur Yvette, France (33(0)1.69.08.62.71; e-mail: guy.cheymol@cea.fr, e-mail: laurent.remy@cea.fr).

A. Gusarov is with SCK $\cdot \mathrm{CEN}$ - Belgian Nuclear Research center, Boeretang 2002400 Mol Belgium (e-mail: andrei.goussarov@sckcen.be). shape after irradiation of the Bragg peak disturb the retrieval of the Bragg wavelength.

The measurements show that for nearly all gratings the Bragg peak remains visible after the irradiation, and that Radiation Induced Bragg Wavelength Shifts (RI-BWSs) vary from few pm (equivalent to an error of less than $1^{\circ} \mathrm{C}$ for a temperature sensor) to nearly $1 \mathrm{~nm}$ (equivalent to $100^{\circ} \mathrm{C}$ ) depending of the FBG types. High RI-BWSs could indeed be expected when considering the huge refractive index variation and compaction of the bare fibre samples that have been measured by other techniques. Post writing thermal annealing is confirmed as a key parameter in order to obtain a more radiation tolerant FBG. Our results show that specific annealing regimes allow making FGBs suitable to perform temperature measurements in a MTR experiment. Index terms- Fiber Bragg Gratting, optical fiber sensors, radiation effect, optical fibres, neutron, gamma, compaction.

\section{REFERENCES}

[1] L. Remy, G. Cheymol, A. Gusarov, A. Morana, E. Marin and S. Girard, "Compaction in optical fibres and fibre Bragg gratings under nuclear reactor high neutron and gamma fluence" IEEE Transactions on nuclear science, Vol. 63, $\mathrm{N}^{\circ} 4$, August 2016.

D. Kinet and P. Mégret are with the Electromagnetism and Telecommunications Department of the University of Mons, 31 Boulevard Dolez, 7000 Mons, Belgium (e-mail: damien.kinet@umons.ac.be).

G. Laffont and T. Blanchet are with the Technological Research Division (TRD) of the French Alternative Energies and Atomic Energy Commission. F91191, Gif sur Yvette, France (e-mail : guillaume.laffont@cea.fr; thomas.blanchet@cea.fr)

T. Blanchet, A. Morana, E. Marin, and S. Girard are with Université de Saint-Etienne, Laboratoire Hubert Curien, UMR CNRS 5516, 18, rue du Pr. Lauras, F-42000 Saint-Etienne, France (e-mails : adriana.morana@univ-stetienne.fr ; emmanuel.marin@univ-st-etienne.fr; sylvain.girard@univ-stetienne.fr). 